\title{
A numerical analysis to investigate the spot weld local influence
}

\author{
Florian Mainnemare ${ }^{1,2, *}$, Benoit Delattre ${ }^{1}$, Ida Raoult $^{1}$, Olivier Villars $^{1}$, Pierre-Alain Boucard ${ }^{2}$, Pierre-Alain Guidault $^{2}$ \\ ${ }^{1}$ PSA groupe, Scientific and Future Technologies Department, 78943 Vélizy-Villacoublay, France \\ ${ }^{2}$ LMT - ENS Cachan - CNRS - Université Paris-Saclay, 61 avenue du Président Wilson, 94235 Cachan, France
}

\begin{abstract}
Relations between existing spot-weld finite element models and the mechanical behaviour of spot-weld are investigated for fatigue design application. Specifically, the different zones that constitute the numerical model are interrogated with respect to physical observation. In order to identify the Mechanical Influence Area of the Spot-Weld here designated as "MIASW" we propose a methodology to determine numerically its size. Hence, we develop a finite element coupon representing a spot-weld under a basis of four unit load cases. The coupon provides mechanical quantities of interest that allows us to characterize the spot-weld response that shell element are unable to represent. This response is crucial to be captured for further fatigue analysis.
\end{abstract}

\section{Introduction}

\subsection{Background}

Existing spot-weld models in literature have been developed for three main applications in the automobile industry. The first one is NVH (Noise, Vibration and Harshness), in a first approximation, it requires accuracy in the structural stiffness and kinematic [8]. The second application is fatigue design; it focuses on different quantities of interest such as the local stiffness and the stress distribution accuracy. The third application is crash analysis and requires nonlinear behavior for large deformation. Its complexity makes it very hard to unify a model with the first two applications, without developing a costly numerical model. However, there are "polyvalent" spot-weld models for both NVH and fatigue applications but those are usually a compromise between the two domains. The challenge is to represent both local and global stiffnesses, within an industrial constraint of minimizing the numerical cost of the model. In our case, although our main objective is to develop such a polyvalent model, we focus in this paper on spot weld models for static purpose and fatigue prediction. In this domain, the existing industrial models commonly represent sheets by shell elements because of their low numerical cost. Hence, we consider as spotweld model all the numerical entities at the spot-weld location that are not part of the sheet model (it can be 1D or 3D element, kinematic coupling, different mechanical behavior).

This study aims to investigate the spot-weld local influence on structural sheet. For that purpose, we develop volumetric finite element models subject to classical in-service mechanical load. We introduce kinematic and static quantities in order to discuss the choices made in the literature. A final objective statement about the relevance in modelling different zones within the model is formulated.

\subsection{Definitions}

Geometrically, the spot-weld nugget is defined as the area delimited by the notch root. However, it has been observed physically that the spot-weld has a metallurgical influence zone on the nearby sheet larger than the nugget, through the Heat Affected Zone (HAZ) [7]. Thus, the spot-weld might affect the local behavior of the structure within a mechanical influence zone as well, through side effects for example. This influence zone could be larger than the nugget. At this point, we define such a zone as the Mechanical Influence Area of the Spot-Weld so-called "MIASW" (see Fig. 1.)

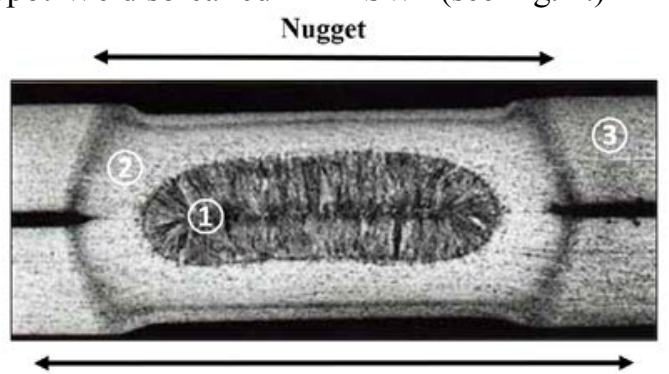

Mechanical Influence Area ?

Fig. 1. Sectional view of a spot-weld for automobile industry purpose. 1/ Coarse grained Heat Affected Zone (HAZ) 2/ SubCritical HAZ 3/ Base metal.[7] The nugget is defined in between the notch roots. The Mechanical Influence Area of the Spot-Weld ("MIASW") cannot be defined geometrically but requires further mechanical analysis.

This raises the necessity to develop an accurate numerical footprint of the spot-weld model that characterizes the mechanical response unable to be 
represented by shell element. We define the Footprint of the Spot-Weld Model ("FSWM" from now on) as the projection area of all numerical entities that constitute the spot-weld model. (see Fig 2.)

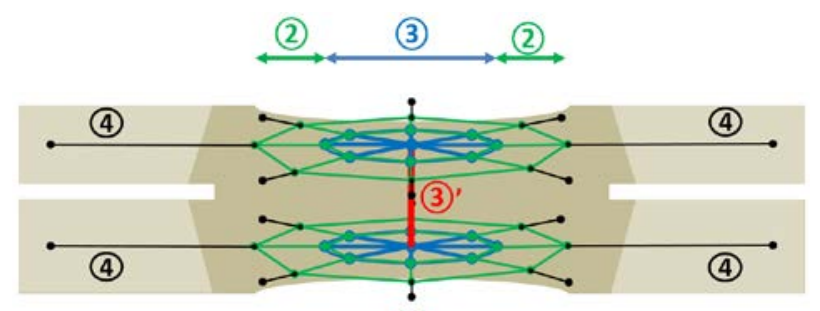

(1)

Fig. 2. Typical recent numerical model of the spot-weld where is represented: 1/ Footprint of the Spot-Weld Model ("FPSWM"). 2/ Peripheral zone. 3 \& 3'/ Both compose the rigid core but could be defined with different numerical parameters. 4/ Shell elements representing the nearby sheet.

We observed so far in literature that the footprint of the existing models was a very scattered data. Indeed, it is mainly used as a calibration parameter to reach the reference stiffness for the joint. And it is mostly evaluated through a volumetric finite element analysis or experimental test. Therefore, the FSWM for fatigue analysis was shown to depend more on the mesh (size and orientation) than on the physical spot-weld influence area. To qualify this specificity we investigate in part 2.1 the choices made around the FSWM in literature. In part 3 we conduct a numerical study in order to estimate the MIASW both statically and cinematically. And thus, what should be the minimum dimension of the FSWM to be able to represent the MIASW. At this point, we could affirm that we need FSWM greater or equal to MIASW in order to represent accurately the mechanical response of the spot-weld that cannot be captured by the shell finite element model. However, we do not investigate here methods that could propose to grant a part of the mechanical accuracy encapsulated inside the spot-weld numerical model. But that would enrich by extrapolation for example, through an off-line additional step, the measured mechanical fields.

Another key point concerns the Rigid Core within the Spot-Weld Model ("RCSWM" from now on). introduced by multiple studies to represent numerically the geometrical stiffness induced by the spot-weld. The dimension of this rigid core is usually justified only through the spot-weld geometry aspect, without additional mechanical investigation to characterize such a behavior. In this study, after recalling in part 2.2 the different divisions between the rigid core and the peripheral zone within the FSWM. We propose in part 3 a method to justify the rigid core dimensions using mechanical quantities of interest.

\subsection{Boundaries}

Our approach is defined in a quasi-static environment with the hypothesis of linear displacement, and linear homogeneous and isotropic material. Justified by the assumption made in fatigue analysis.
Furthermore, we consider an academic problem with the assumption of no geometrical perturbation in certain vicinity of the spot-weld.

\section{State of the art around spot-weld models}

\subsection{Existing dimensions of FSWM}

We decided to distinguish the existing FSWM with respect to their dimension relatively to the nugget dimension, as it is a clear measurable entity. We distinguished three categories: no footprint, a footprint equal to the nugget and larger than the nugget.

\subsubsection{No FSWM}

The first group gathers the so-called Point to Point (P2P) models, joining the sheets through a $1 \mathrm{D}$ beam or rigid element. It results in a much localized influence area of the link. The beam parameter as the diameter, the second moment of inertia, the Young modulus are usually calibrated in order to match the global stiffness of volumetric [4] or experimental reference loading. These models involve an erroneous local stress distribution, overcome by analytical stress function or volumetric refined model associated to the loading case of the joint [9]. Moreover, a major drawback of this method concerns the numerical processing for the mesh congruent requirement.

\subsubsection{FSWM equal to the nugget}

The second group includes the models defined with a FSWM taken equal to the spot-weld nugget diameter. This connection area is usually defined with no more justification than geometry even if the axial symmetry of the nugget is not respected [10].

\subsubsection{FSWM larger than the nugget}

The third group collects the models with a FSWM larger than the spot-weld nugget diameter. For most of them, the extra area allows the spot weld model to transmit bending rigidity to the sheet. They use Rigid Body Element (RBE), kinematic coupling or shell elements to define the contact area [1]. The stiffness calibration is commonly based on the mechanical response of a volumetric structure or an experimental test. [13], [2]. The model developed by [6] could be considered to affect a larger zone as well as it uses eXtended FiniteElement to improve the spot-weld kinematic through analytical displacement functions spread below the nugget. 


\subsection{Rigid core inclusion}

For geometrical reasons, the structural stiffness of the spot-weld nugget is higher than the rest of the structure. Hence, in the literature a large diversity exists in the modelling of this phenomenon. It appears that different material behaviors are sometimes attributed to the different regions of the spot-weld model. In regards to this distinction, we could classify the existing models into three categories.

\subsubsection{Rigid core and elastic peripheral zone}

The first category distinguish an infinitely rigid core on the one side and a peripheral elastic zone on the other side. It is for example the case for the Spider model where a central part is composed of a rigid element to link the facing sheets and 16 beam elements connected to the surrounding shell elements. We found a few comparable models in the literature but less widely used for industrial applications [12]. Similarly, the "umbrella" or "ACM1" model is made of a rigid central element and beam elements connect the core to the sheets [11]. In the same way, the Bolt Spot model developed by Renault is composed of a stiff beam and a shell core with adjustable properties (thickness and/or Young modulus) possibly different from the regular shell elements describing the sheets [3].

\subsubsection{Elastic core and peripheral zone}

We could introduce a second category, which distinguishes a spot-weld core and a peripheral zone, but eventually characterized by different behaviors. For example the "ACM2" model developed by (Heiserer et $\mathrm{Al})$ for the convenience of numerical implementation is made of a solid elastic hexahedral element to characterize the spot-weld core and is connected to the rest of the sheet through rigid body elements. The MSC Cweld model developed by NASTRAN software enters this category as well. It is an elastic beam connected to the two sheets through patches with local kinematical constraint.

\subsubsection{No peripheral zone}

Finally, a last category can be introduced for the models with no distinction between a spot-weld core and a zone of influence. The early developed and low cost "P2P" models are part of this category The "single brick" model does not make any area distinction neither. It is defined as a hexahedral element connecting two facing elements of both sheets. It requires a congruent mesh as well[10]. The model developed by[13] is characterized by a complete rigid zone linked to the surrounding shell elements of the structure. Although it is enhanced with analytical stress distribution function, no differentiation of area is proposed. Similarly, the model developed by [5] extends the linking area with analytical displacement function but does not explicitly distinguish a central part from a crown in the spot-weld model.

\section{Method}

In our study we first investigate whether the Footprint of the Spot-Weld Model ("FSWM") could be related to the Mechanical Influence Area of the Spot-Weld ("MIASW"). For that purpose, we use a fully volumetric finite element mesh of a circular coupon. This model is made of two sheets linked at their center by a spot-weld. We apply four unit loading cases to the sheet edges. Then, we extract both kinematic and static quantities in order to conclude on the size of the MIASW. As a second step, we question how to determine the size of the RCSWM. We proposed a method of determination of this zone through kinematic and stress distribution analyses.

\subsection{Coupon characteristics}

\subsubsection{Determination of the MIASW}

For the coupon dimensions, we elected a representative case of what could be found in an industrial context. The coupon has a total diameter of $34 \mathrm{~mm}$ for a $6 \mathrm{~mm}$ nugget diameter at the centre. This is based on the typical minimal distance from a spot-weld to another. Upper and lower sheet are respectively $1.5 \mathrm{~mm}$ and $1.3 \mathrm{~mm}$ thick, with a $0.2 \mathrm{~mm}$ inter-sheet length. The nugget diameter is $6 \mathrm{~mm}$. Material parameters are taken equal to the one used for the sheet metal structure. The finite element model is constituted of 60096 solid quadratic hexahedral elements of type C3D20 in Abaqus/Standard (Version 6.16, Simulia Corp., RI, USA). It results in a little more than 1.5 million of degrees of freedom. The radial mesh size is $1 \mathrm{~mm}$ until the spot-weld radius at which it is refined to $0.2 \mathrm{~mm}$. The mesh is axisymmetric above a radius of $0.1 \mathrm{~mm}$. Finally, each sheet is made of 8 elements within the thickness.

\subsubsection{RCSWM}

The same numerical model is used for this part except that a kinematic coupling is defined between all the nodes within a cylindrical central volume. This cylindrical central volume shares the same axis as the coupon, with a variable diameter and a height from the top surface to the bottom surface of the coupon.

\subsection{Boundary conditions}

Boundary conditions are defined identically for the two analyses. 


\subsubsection{Loading of the coupon}

The following procedure is defined in order to avoid any residual load in the loading zone area.

For both the upper and lower sheet individually (see Fig 3.a), b) )., we define a kinematic coupling constraint between a central geometrical point and all the nodes at the radius: $\mathrm{R}=\mathrm{R}_{\text {Coupon. }}$. The two geometrical points lie at the same position: the intersection of the coupon axis and the middle plane of the inter-sheet. But they undergo different couplings. Structural loading is applied through the point coupled to the upper sheet. While the dual degree of freedom of the point coupled to the lower sheet is set equal to zero. The constrained degree of freedom through the kinematic coupling is defined as it is opposed to the loading.

Rigid body movements are locked up through isolated nodes $\left(\mathrm{N}_{\mathrm{x}}\right.$ and $\left.\mathrm{N}_{\mathrm{y}}\right)$ with respect to a minimum additional boundary conditions (see Fig 3.) The resulting loads within these isolated nodes are verified to be close to zeros in order to confirm that they lock only rigid body movement without inducing interfering loads.

a)

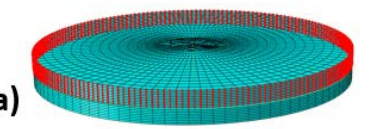

b)

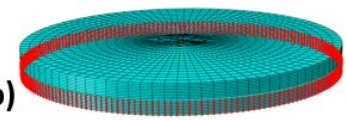

c)
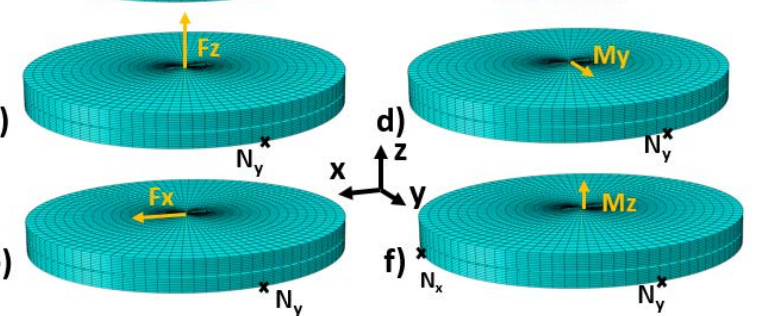

e)

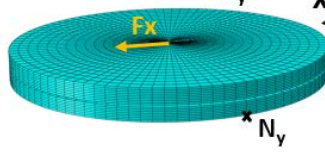

Fig. 3. Coupon boundary conditions and loading cases of the coupon. a) Upper crown connected to geometrical center point. b) Lower crown connected to geometrical center point. c) Tensile loading case in $\mathrm{z}$ direction (Fz). d) Bending loading case in $\mathrm{y}$ direction (My). e) Shear loading case in $\mathrm{x}$ direction $(\mathrm{Fx}) . \mathrm{f})$ Torsion loading case in $\mathrm{z}$ direction $(\mathrm{Mz})$

\subsection{Quantities of interest}

In this part, we introduce mechanical quantities of interest in order to be able to quantify the MIASW. The aim is to investigate in which area the necessary conditions for the shell theory to apply are not satisfied. Thus, we decide to focus on two major necessary conditions of the shell theory application. The first one is based on the kinematic hypothesis for shell theory for both Kirchhoff-Love and Reissner-Mindlin cases. It stipulates that every fiber normal to the mean surface of the shell remains straight after deformation. The second hypothesis states that stress distribution is planar. More precisely, regarding the Kirchhoff-Love shell theory definition, out-of-plane components of the stress tensor are negligible compared to the in-plane stress components. Thus, the stress tensor becomes:

$$
\underline{\underline{\boldsymbol{\sigma}_{K L}}}=\left[\begin{array}{ccc}
\sigma_{x x} & \sigma_{x y} & 0 \\
& \sigma_{y y} & 0 \\
s y m & & 0
\end{array}\right]
$$

Differently, for the Reissner-Mindlin theory, the out-ofplane shear stress is taken into account. It leads us to:

$$
\underline{\underline{\boldsymbol{\sigma}_{\boldsymbol{R}}}}=\left[\begin{array}{ccc}
\sigma_{x x} & \sigma_{x y} & \sigma_{x z} \\
& \sigma_{y y} & \sigma_{y z} \\
s y m & & 0
\end{array}\right]
$$

In order to evaluate the validity of these conditions, we introduce the following quantities of interest (4) \& (5).

\subsubsection{Kinematic hypothesis validity}

The coupon is meshed to obtain aligned nodes along the thickness of the sheets. Therefore on the deformed model, we are able to compute the linear regression of the fibres normal to the mean plane for each sheet. We designate them as "numerical thickness fibres" as they are the consequence of the numerical finite element modelling. The linear fitting regression is carried out through the Principal Components Analysis method. Then we are able to extract the equation of the best linear fit and indicators about the quality of the linear regression (disparity, standard deviation, Pearson's correlation coefficient). We decide to elect a first order statistical indicator to represent with clarity the deviation between the thickness fibre and its linear fit. Hence, we introduce the distance " $\mathrm{d}$ " between the numerical thickness fibre and its linear regression under the following definition: it is the distance between a point within the thickness fibre and its orthogonal projection on the linear (see figure 4.). In the following, we will thus quantify the amplitude of the buckling of the normal fibre through the distance quantity $\delta$ :

$\forall j \in\left[1, \ldots, N_{\text {Fibres }}\right], \quad \delta_{j}=\max \left\{d_{i}, i=\left[1, \ldots, N_{\text {nodes }}^{j}\right]\right\}$

Where $N_{F i b r e s}$ is the number of fibres of the coupon and $N_{\text {nodes }}^{j}$ is the number of nodes of fibre $\mathrm{j}$.

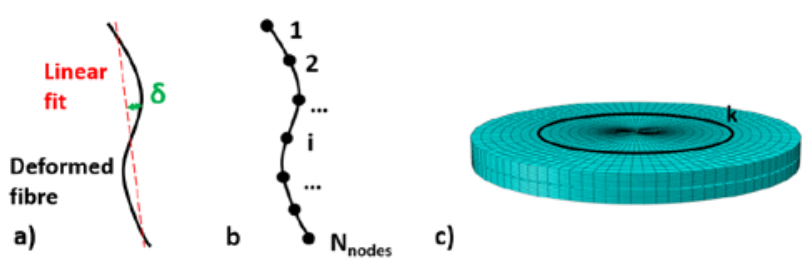

Fig. 4. a) Measure of the distance between a deformed thickness fibre and its linear fit. b) Nodes distribution along a thickness fibre $j$ included in a fixed radius $k$. c) fixed radius $k$ of the upper sheet 


\subsubsection{Static hypothesis validity}

In order to evaluate the validity of the planar stress hypothesis, we need to introduce a quantity of interest that indicates the amplitude ratio between in-plane and out-of-plane stresses. Thus for every thickness fibre at a fixed radius, we evaluate the value of the highest ratio between the maximum out-of-plane stress to the maximum in-plane stress of each node of the thickness fibre. In other terms, the stress ratio $S_{O P}$ is defined as:

$$
S_{O P}=\max _{j}\left(\max _{i}\left[\frac{\sigma_{z Z}^{i j}}{\sigma_{I P \text { maxi }}^{i j}}\right]\right)
$$

Where $\sigma_{z z}^{i j}$ and $\sigma_{I P \max i}^{i j}$ are respectively the $\sigma_{z z}$ component of the stress tensor and the maximum InPlane stress components. For the node $i$ belonging to the thickness fibre $j$ (see Fig 4.b).

Similarly, we introduce:

$$
S_{O P S}=\max _{j}\left(\max _{i}\left[\frac{\sigma_{O P S \max i}^{i j}}{\sigma_{I P \max i}^{i j}}\right]\right)
$$

Where $\sigma_{O P S \text { maxi }}^{i j}$ is the maximum Out-of-Plane stress component of the node $i$ belonging to the thickness fibre $j$ (see Fig 4.b). Including the Out-ofPlane Shear (OPS) stress components. Hence, we could evaluate $S_{O P}$ and $S_{O P S}$ for each radius along the coupon.

\section{Results}

\subsection{Mechanical quantities of interest for MIASW estimation}

\subsubsection{Kinematic hypothesis validation}

In this part we aim at evaluating the area around the spot weld where the kinematic hypothesis of a thickness fibre remaining straight during deformation is not respected. First, we plot the evolution of the distance quantity $\delta$ along the coupon, for every loading case.
Distance from notch root (in thickness of sheet)
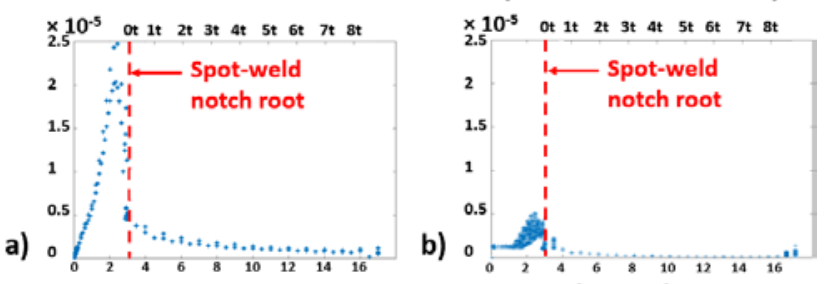

Distance from coupon center (in $\mathrm{mm}$ )

Distance from notch root (in thickness of sheet)
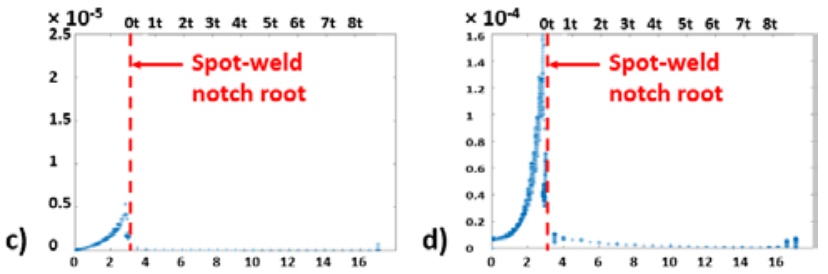

Distance from coupon center (in $\mathrm{mm}$ )

Fig. 5. Amplitude of the quantity of interest $\delta$, according to the radial distance to the middle of the coupon. Radial distance is both expressed in millimetres and in thickness of the sheet unity. The response is given for the four load cases: a) tensile load; b) bending load; c) torsion load; d) shearing load.

As a first observation, the phenomenon seems to have a relatively small order of magnitude. Indeed, we observe that the shear loading case induce the largest thickness fibre buckling. For that case the quantity $\delta$ reaches a maximum of $1.610^{-4} \mathrm{~mm}$ of deformation for a $1.5 \mathrm{~mm}$ thick sheet. The thickness fibre buckling is mostly localized within the nugget area and drops quickly beyond the spot-weld notch root. We decide to analyse the buckling relatively to the global deformation of the coupon. Thus, we plot (Fig 6.) the evolution of the ratio between the quantity of interest $\delta$ and the maximum displacement of the structure for each loading case.

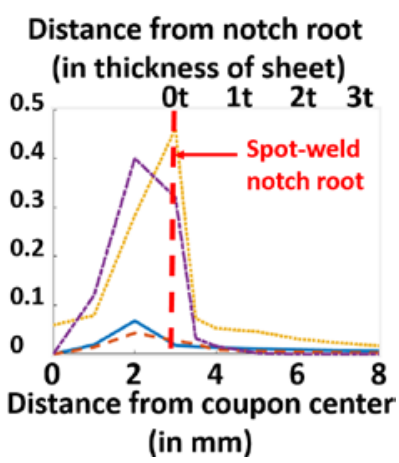

Fig. 6. Ratio (in \%) between the quantity of interest $\delta$ to the maximum displacement of the structure each loading case. Amplitude of the quantity of interest $\delta$, according to the radial distance to the middle of the coupon. Radial distance is both expressed in millimetres and in thickness of the sheet unity. The response is given for the four loading cases:

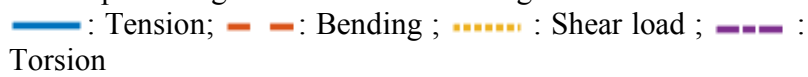

The ratio is found to reach at maximum $0.46 \%$ for the shear load but drops to $0.05 \%$ at a distance of one sheet thickness from the spot-weld notch root. For both tensile, 
bending and torsion loads, the ratio beyond one time the sheet thickness from the notch root is lower than $0.01 \%$.

\subsubsection{Static hypothesis validation}

Similarly to the kinematic approach, we aim to evaluate in which area around the spot-weld the hypothesis of planar stress distribution is not respected. For that purpose, we plot in Fig 7. the evolution of the stress ratios $S_{O P}$ (Fig 7.a) \& b) ) and $S_{O P S}$ (Fig 7.c) \& d) ) along the coupon, for every loading case.

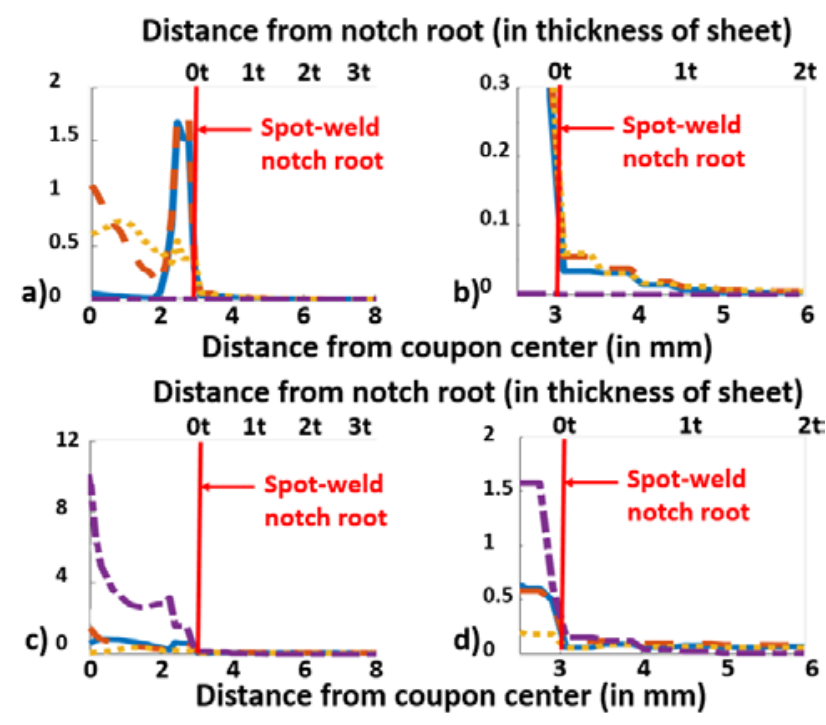

Fig. 7. a) \& b): Amplitude of the stress ratios $S_{O P}$ for each loading case according to the radial distance to the middle of the coupon. b) is simply a zoom of a)

c) \& d): Amplitude of the stress ratios $S_{O P S}$ for each loading case according to the radial distance to the middle of the coupon. d) is simply a zoom of c)

$\underline{-}$ : Tensile load; -- : Bending load ; $\ldots \ldots \ldots$ : Shearing load ; --ー : Torsion load

Radial distance is both expressed in millimetres and in thickness of the sheet unity.

We need to analyse separately the two stress quantities of interest.

The stress ratio amplitude of $S_{O P}$ drops drastically beyond the spot-weld notch root. At one time the sheet thickness it is equal to $0.01 \pm 0.004$ for the tensile, bending and shearing loading cases. For the torsion load this ratio is equal to $1.710^{-5}$. Beyond one time the sheet thickness, $S_{O P}$ decrease until reaching zero. For example it is equal to $7.210^{-7} \pm 0.110^{-7}$ at three times the sheet thickness beyond the notch root.

The stress ratio amplitude of $S_{O P S}$ drops drastically beyond the spot-weld notch root as well. However, at one time the sheet thickness beyond the notch root the stress ratio amplitude is found to be equal to $0.081 \pm 0.016$ for the tensile, bending and shearing loading cases. For the torsion loading case, this ratio is equal to 0.02 . Furthermore, when moving away to three times the sheet thickness beyond the notch root, the stress ratio $S_{O P S}$ is found to be equal to $0.052 \pm 0.014$ for the tensile, bending and shearing loading cases. It remains constant until the end of the coupon which is six times the sheet thickness beyond the notch root. For torsion, the stress ratio $S_{O P S}$ is found to remain close to zero from two times the sheet thickness beyond the notch root where it is equal to $1.310^{-5}$.

\subsection{Mechanical quantities of interest for RCSWM estimation}

The aim of this part is to evaluate the equivalent size of the RCSWM that represents the mechanical response of the spot-weld correctly. For that purpose, we consider the coupon defined in part 3.1.1 as a numerical reference of the coupon defined in part 3.1.2. Then, the loading cases described in part 3.2.1 are applied. The aim is to find the rigid central cylinder that leads to a similar kinematic and load distribution than the reference coupon. Once the rigid core area is modelled with rigid elements, the numerically elected MIASW has to remain unchanged. Results for this study are expected soon to conclude on this topic.

\section{Conclusion}

In this study, we first raise a summary of the existing spot-weld models for fatigue design, in the automotive industry.

Then we describe a methodology to evaluate the dimension of the MIASW. We evaluate the area in which hypothesis of the shell theory are not respected. It allows us to give a numerical justification on the dimension of the MIASW. We are able to conclude that, with respect to the Reissner-Mindlin, thick shell theory, the MIASW lies approximately until one sheet thickness beyond the notch root of the spot-weld. Beyond this area, the shell theory hypotheses are validated. However, the results we obtained lead to a different conclusion if Kirchhoff-Love shell theory is used for shell modelling. In the far vicinity of the spot-weld (up to six times the sheet thickness beyond the notch root) the planar stress distribution hypothesis appears to be less clearly respected. The maximum Out-of-Plane stress amplitude represents $5 \%$ of the In-Plane maximum stress amplitude.

In literature, RCSWM is found to lead to different modelling within different areas of application. We introduce a methodology that could characterize its dimension with respects to mechanical quantities of interest. However, results concerning the investigation on the RCSWM dimension remain to be expected soon. It will make it possible to model this area with rigid elements for example, reducing the numerical cost of the model.

Finally, with this study we were able to propose a MIASW area of the spot-weld. However, the rest of the MIASW needs to be properly modelled in order to characterize the mechanical fields necessary for fatigue analysis. Indeed we know that this region of interest is 
crucial for fatigue analysis. Thus, the topic will be largely covered in further studies.

\section{Bibliography}

[1] MSC.Nastran 2003: Linear Static Analysis Users Guide, MSC.Software Corporation, Santa Ana, CA 92707 USA.

[2] J. Andersson and J. Deleskog. Fatigue life and stiffness of the spider spot weld model. Master's thesis, Chalmers University of Technology, 2014.

[3] Y. Baik. spot weld modeling for vehicule durability performance with ansa. In 4th ANSA \& META International Conference, 2011.

[4] J-F. Beniguel. Modélisation du comportement mécanique des assemblages de structures soudées. $\mathrm{PhD}$ thesis, Cachan, Ecole normale supérieure, 2002.

[5] P. Burry. Quelques techniques d'analyse mécanique pour les structures automobiles. $\mathrm{PhD}$ thesis, Ecole Polytechnique, 2007.

[6] P. Burry, M. Habibou, and G. Billotey. Elémentsfinis étendus pour la modélisation des structures soudées par points. Mechanics \& Industry, 9(2):103-108, 2008.

[7] S. Dancette, D. Fabregue, V. Massardier, J. Merlin, T. Dupuy, and M. Bouzekri. Experimental and modeling investigation of the failure resistance of advanced high strength steels spot welds. Engineering Fracture Mechanics, 78(10):2259-2272, 2011.

[8] RO. De Alba Alvarez, NS. Ferguson, and BR. Mace. A robust spot weld model for structural vibration analysis. Finite Elements in Analysis and Design, 89:17, 2014.

[9] F. Jean-Luc. Dimensionnement à la fatigue polycyclique de structures soudées. $\mathrm{PhD}$ thesis, Palaiseau, Ecole polytechnique, 1996.

[10] K. Pal and D.L. Cronin. Static and dynamic characteristics of spot welded sheet metal beems. Transactions of the ASME, 117:316-322, 1995.

[11] M. Palmonella, M. I. Friswell, J. E. Mottershead, and Lees A. W. Finite element models of spot welds in structural dynamics: review and updating. Computers and Structures, 83 (8-9):648-661, 2005.

[12] M. M. Rahman, R. A. Bakar, M. M. Noor, M.R.M Rejab, and MSM. Sani. Fatigue life prediction of spotwelded structures: a finite element analysis approach. European Journal of Scientific Research, 22(3):444456, 2008.

[13] P. Salvini, E. Scardecchia, and F. Vivio. Fatigue life prediction on complex spot welded joints. SEA 1997 Trans J Mater Manufact, 106:967-75, 1997. 